\title{
SPRR3, a novel miR-338-3p target, regulates the malignant progression of clear cell renal cell carcinoma in vitro via the PI3K/Akt signaling pathway
}

\author{
MAN WU ${ }^{1}$, QIAOYAN GUO ${ }^{1}$, XIANJUN LIU ${ }^{2}$ and LINLIN WU ${ }^{1}$ \\ ${ }^{1}$ Department of Nephrology, The Second Hospital of Jilin University, Changchun, Jilin 130041; \\ ${ }^{2}$ College of Food Engineering, Jilin Engineering Normal University, Changchun, Jilin 130052, P.R. China
}

Received August 15, 2021; Accepted February 10, 2022

DOI: $10.3892 /$ etm.2022.11246

\begin{abstract}
Clear cell renal cell carcinoma (ccRCC) is the most common type of renal cell carcinoma and has a poor prognosis. However, its underlying mechanisms remain unclear. The present study aimed to evaluate the role of small proline-rich repeat protein 3 (SPRR3) in the proliferation, migration and invasion of ccRCC cells and to investigate its upstream and downstream regulatory mechanisms. Survival analysis was performed using the UALCAN website based on the The Cancer Genome Atlas database. Normal renal cell line HK-2 and ccRCC cell lines (786-O, CaKi-1 and UMRC-2) were used. Reverse transcription-quantitative PCR (RT-qPCR) was used to detect mRNA and microRNA (miRNA) levels. Western blotting was used to detect protein levels. Cell Counting Kit-8 and colony formation assays, a wound healing assay and a Transwell invasion assay were used to assess the proliferation, migration and invasion of ccRCC cells, respectively. Transfection of overexpression plasmids and small interfering RNAs were used to upregulate and knockdown SPRR3 expression, respectively. Transfection of miRNA-mimics was used to overexpress miR-338-3p. A luciferase reporter gene assay was used to verify the predicted binding relationship between SPRR3 mRNA and miR-338-3p. The results indicated the following: i) SPRR3 was a risk factor for the survival of patients with ccRCC, and was upregulated in ccRCC cell lines; ii) SPRR3 promoted the proliferation, migration and invasion
\end{abstract}

Correspondence to: Professor Linlin Wu, Department of Nephrology, The Second Hospital of Jilin University, 218 Ziqiang Street, Changchun, Jilin 130041, P.R. China

E-mail:wull@jlu.edu.cn

Dr Xianjun Liu, College of Food Engineering, Jilin Engineering Normal University, 3050 Kaixuan Street, Changchun, Jilin 130052, P.R. China

E-mail: liuxianjun@jlenu.edu.cn

Key words: clear cell renal cell carcinoma, small proline-rich repeat protein 3, microRNA-338-3p, PI3K/Akt signaling pathway, phenotypes of ccRCC cells; iii) SPRR3 regulated the tumor phenotypes of ccRCC cells via the PI3K/Akt pathway; iv) miR-338-3p directly targeted SPRR3 mRNA and negatively regulated SPRR3 expression; and v) miR-338-3p inhibited the PI3K/Akt pathway and the tumor phenotypes of ccRCC cells by downregulating SPRR3. In conclusion, SPRR3, as a novel target of miR-338-3p, regulated the proliferation, migration and invasion of ccRCC cells via the PI3K/Akt pathway; this finding not only enriches our understanding of the mechanism underlying ccRCC development, but also demonstrates a potential novel therapeutic target for this disease.

\section{Introduction}

Renal cell carcinoma ( $\mathrm{RCC}$ ) is the most fatal genitourinary malignant tumor. Clear cell renal cell carcinoma (ccRCC) is the most common type of RCC (1); however, the underlying mechanism remains unclear. Although treatments for ccRCC have been explored for years, there are no available approaches that provide satisfactory results (2). The 5-year survival rate of patients with advanced tumors is only $23 \%$ (3). Therefore, there is a need to uncover the molecular mechanisms underlying the pathogenesis of ccRCC and identify novel therapeutic targets.

Small proline-rich repeat protein 3 (SPRR3) is a member of the small proline-rich protein family. SPRR3 has been associated with the progression of multiple cancer types. SPRR3 was reported as a tumor promoter in colorectal cancer, breast cancer, glioblastoma multiforme and non-small-cell lung cancer (4-7), while in esophageal cancer, it was revealed to be a tumor suppressor (8). It also plays a role in non-tumor cells. SPRR3 acts to promote cell survival in vascular smooth muscle cells (9), and proliferation and matrix synthesis of cardiac fibroblast (10). However, the role of SPRR3 in ccRCC remains to be elucidated. In previous years, microRNA (miRNA) dysfunction has been revealed to be involved in the pathogenesis of various cancer types (11). MiRNAs can downregulate target gene expression by specifically binding to the 3'untranslated region (3'UTR). miR-338-3p inhibits the proliferation, migration and invasion of ccRCC cells (12-14); however, the underlying mechanism remains unclear, and to the best of our knowledge no study has reported the association between miR-338-3p and SPRR3. Activation of 
the PI3K/Akt signaling pathway promotes ccRCC progression (15). Furthermore, this pathway mediates the regulation of RCC cells by miR-338-3p (16). However, it remains unclear whether this pathway is regulated by SPRR3 in ccRCC cells.

The present study aimed to evaluate the relationship between SPRR3 expression and ccRCC prognosis and to detect its expression in the normal human renal cell line HK-2 and ccRCC cell lines. Furthermore, the current study aimed to evaluate the roles of SPRR3 in the tumor phenotypes of 786-O cells, including their proliferation, migration and invasion. Notably, this study aimed to further investigate the upstream and downstream regulatory mechanisms of SPRR3.

\section{Materials and methods}

Bioinformatics analysis. UALCAN (http://ualcan.path.uab. edu/) (17), a web portal for analyzing cancer data (project ID: TCGA-KIRC) from The Cancer Genome Atlas TCGA database (18), was used to assess the relationship between SPRR3 expression and prognosis in patients with ccRCC. The public prediction platform TargetScan (version 7.2; http://www. targetscan.org/vert_72/) was used to predict the potential miRNAs those target the 3'UTR of SPRR3 mRNA (19).

Cell culture. HK-2 (cat. no. SCSP-511), 786-O (cat. no. TCHu186) and CaKi-1 (cat. no. TCHu135) cell lines were purchased from the National Collection of Authenticated Cell Cultures (Shanghai China), whereas the UMRC-2 cell line (cat. no. HTX2941) was purchased from Otwo Biotech Co., Ltd. The cells were cultured in Dulbecco's modified Eagle's medium (DMEM) containing 10\% fetal bovine serum (FBS) and $1 \%$ penicillin/streptomycin at $37^{\circ} \mathrm{C}$, under a humidified atmosphere of $5 \% \mathrm{CO}_{2}$. For each cell line, three replicates were obtained using a parallel culture for subsequent experiments $(n=3)$. All cell culture reagents were obtained from Gibco (Thermo Fisher Scientific, Inc.).

Reverse transcription-quantitative PCR (RT-qPCR). Total RNA was isolated from each sample (HK-2, 786-O, CaKi-1 and UMRC-2 cell lines) using TRIzol ${ }^{\circledR}$ reagent (Invitrogen; Thermo Fisher Scientific, Inc.). cDNA for SPRR3 and $\beta$-actin (ACTB) detection were synthesized using the TransScript All-in-One First-Strand cDNA Synthesis SuperMix (Beijing Transgen Biotech Co., Ltd.) following the manufacturer's instructions, while qPCR was performed using TransStart ${ }^{\circledR}$ Top Green qPCR SuperMix (Beijing TransGen Biotech Co., Ltd.). A TaqMan miRNA assay kit (Ambion; Thermo Fisher Scientific, Inc.) was used for the synthesis of cDNA for miR-338-3p and U6 detection and RT-qPCR analysis. The reaction was carried out using the following parameters: Initial denaturation at $94^{\circ} \mathrm{C}$ for $10 \mathrm{~min}$; 40 cycles of denaturation at $94^{\circ} \mathrm{C}$ for $5 \mathrm{sec}$; annealing at $60^{\circ} \mathrm{C}$ for $15 \mathrm{sec}$; extension at $72^{\circ} \mathrm{C}$ for $10 \mathrm{sec}$; and dissociation. RT-qPCR was performed using a LightCycler ${ }^{\circledR} 480$ system (Roche Diagnostics). SPRR3 and miR-338-3p levels were normalized to those of ACTB and U6, respectively. The primers of miR-338-3p and U6 were used following a previous study (20), and both reverse primers are universal. The $2^{-\Delta \Delta C q}$ method was used to calculate the relative mRNA expression levels of these genes (21). The primers used for qPCR are listed in Table I. Each sample was run in triplicates.
Antibodies and drugs. The primary antibodies used for western blotting were as follows: Anti-SPRR3 (1:1,000; cat. no. DF12751; Affinity Biosciences, Ltd.), anti- $\beta$-actin $(1: 2,500$; 60008-1-Ig; Proteintech Group, Inc.), anti-phospho-pan-Akt (1:500; cat. no. AF0016; Affinity Biosciences, Ltd.), and anti-pan-Akt (1:500; cat. no. AF6261; Affinity Biosciences, Ltd.). The secondary HRP-conjugated antibodies (1:5,000; goat anti-mouse, SA00001-1; goat anti-rabbit, SA00001-2; Proteintech Group, Inc.) were used for western blotting. Recombinant human insulin-like growth factor-1 (IGF-1) protein (cat. no. 291-G1; R\&D systems, Inc.), an agonist of the PI3K/Akt pathway, was dissolved in DMEM and used at a concentration of $100 \mathrm{ng} / \mathrm{ml}$.

Western blotting. The cells were harvested and processed with RIPA lysis buffer (CST Biological Reagents Co., Ltd.) supplemented with phenylmethylsulphonyl fluoride (Thermo Fisher Scientific, Inc.), protease inhibitor cocktail (TransGen Biotech Co., Ltd.) and phosphatase inhibitor cocktail (TransGen Biotech Co., Ltd.). Western blotting was performed as described below, and the protein samples $(20 \mu \mathrm{g})$ were separated on $10 \%$ gels using SDS-PAGE, and then electro-transferred onto PVDF membranes (Immobilon-P; MilliporeSigma). After blocking with $5 \%$ bovine serum albumin (MilliporeSigma) in Tris-buffered saline containing $0.05 \%$ Tween 20 for $1 \mathrm{~h}$ at room temperature, the PVDF membranes were incubated overnight with the primary antibodies at $4^{\circ} \mathrm{C}$. Following incubation with the corresponding secondary antibodies for $1 \mathrm{~h}$ at room temperature, protein chemiluminescence was detected using the BeyoECL Plus kit (Beyotime Institute of Biotechnology) in a KETA GL Imaging System (Wealtec Corp.), and the gray value of the band was quantified using ImageJ (version 1.51; National Institutes of Health). $\beta$-actin was used as the control.

Cell transfection. Small interfering RNAs (siRNAs) for SPRR3 knockdown and control-siRNAs (scrambled siRNAs) were purchased from Sangon Biotech Co., Ltd. with the sequences listed in Table II. miR-338-3p-mimics (cat. no. MC10716) for miR-338-3p overexpression and the corresponding control-mimics (cat. no. 4464058) were purchased from Thermo Fisher Scientific, Inc. The human SPRR3 gene (accession no. NM_005416.3) was cloned into pcDNA 3.1/His B (cat. no. V385-20; Thermo Fisher Scientific, Inc.) for protein overexpression (Fig. S1), and the empty vector was transfected as a control. Lipofectamine 3000 transfection reagent (L3000075; Thermo Fisher Scientific, Inc.) was used to transfect these plasmids $(1 \mu \mathrm{g} / \mathrm{ml})$, siRNAs $(50 \mathrm{nM})$ and miRNA-mimics $(50 \mathrm{nM})$ into $786-\mathrm{O}$ cells following the manufacturer's instructions. After transfection at $37^{\circ} \mathrm{C}$ for $6 \mathrm{~h}$, the culture medium was replaced with fresh medium and cells were incubated at $37^{\circ} \mathrm{C}$ for another $24 \mathrm{~h}$.

Cell Counting Kit-8 (CCK-8) assay. A CCK-8 assay was used to assess the proliferation of 786-O cells. Viable cell counts were indirectly determined by measuring optical density (OD) values. The cells were seeded at $3 \times 10^{3}$ cells per well in a 96-well culture plate, excluding the use of external rows and columns to avoid the edge effects. After complete cell attachment, the cells were processed according to the experimental requirements. When needed, IGF-1 was used at a concentration 
Table I. Sequences of primers used for reverse transcription-quantitative PCR.

\begin{tabular}{lll}
\hline Gene & \multicolumn{1}{c}{ Forward $\left(5^{\prime}-3 '\right)$} & \multicolumn{1}{c}{ Reverse $\left(5^{\prime}-3^{\prime}\right)$} \\
\hline SPRR3 & CTTCTCTGCACAGCAGGTCC & AGCAATTTAATGAGGGAAGAGC \\
ACTB & CTCCATCCTGGCCTCGCTGT & GCTGTCACCTTCACCGTTCC \\
miR-338-3p & TGCGGTCCAGCATCAGTGAT & CCAGTGCAGGGTCCGAGGT \\
U6 & GCTCGCTTCGGCAGCACA & GAGGTATTCGCACCAGAGGA
\end{tabular}

SPRR3, small proline-rich repeat protein 3; ACTB, $\beta$-actin; miR, microRNA.

Table II. Sequences of siRNAs.

\begin{tabular}{lll}
\hline siRNA & \multicolumn{1}{c}{ Forward (5'-3') } & Reverse (5'-3') \\
\hline Control-siRNA & UUCUCCGAACGUGUCACGUTT & ACGUGACACGUUCGGAGAATT \\
SPRR3-siRNA-1 & CUGAAUUAAGCAGAAAGUCUUTT & AAGACUUUCUGCUUAAUUCAGTT \\
SPRR3-siRNA-2 & CCCAUCUGUUUCUGUGUCUUATT & UAAGACACAGAAACAGAUGGGTT
\end{tabular}

siRNA, small interfering RNA; SPRR3, small proline-rich repeat protein 3.
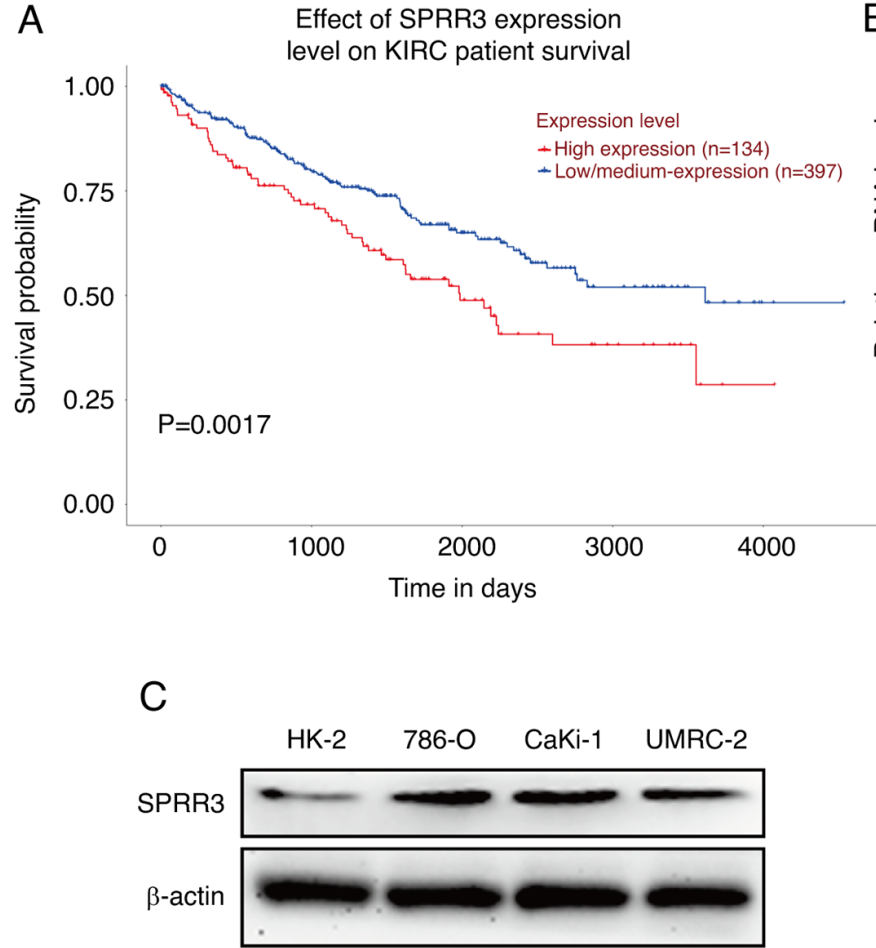
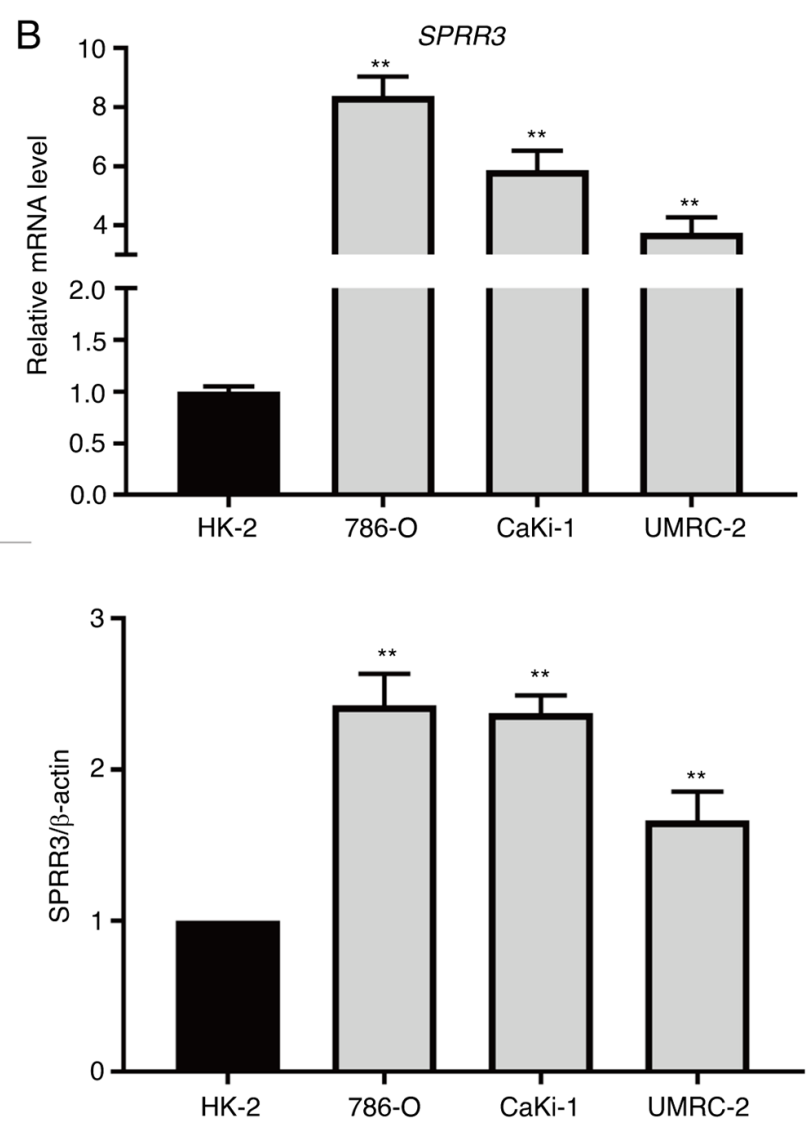

Figure 1. Association between SPRR3 expression and the prognosis of ccRCC, and detection of SPRR3 expression in human normal renal cells HK-2 and ccRCC cell lines. (A) Survival analysis of 531 patients with ccRCC by Kaplan-Meier curve (P=0.0017). SPRR3 expression in HK-2, 786-O, CaKi-1 and UMRC-2 cell lines, as detected using (B) reverse transcription-quantitative PCR and (C) western blotting. ${ }^{* *} \mathrm{P}<0.01$ vs. HK-2. SPRR3, small proline-rich repeat protein 3; ccRCC, clear cell renal cell carcinoma.

of $100 \mathrm{ng} / \mathrm{ml}$. After treatment for $0,24,48$ and $72 \mathrm{~h}, 10 \mu \mathrm{l}$ of CCK-8 (APExBIO Technology LLC) solution was added to each well. After incubation for $2 \mathrm{~h}$ at $37^{\circ} \mathrm{C}$, the absorbance was measured at $450 \mathrm{~nm}$ using a microplate reader (Thermo Fisher Scientific, Inc.). The CCK-8 assay results were expressed as OD values. 
A

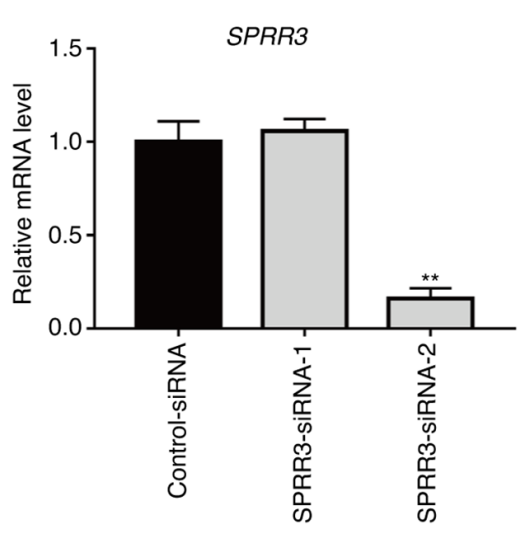

D
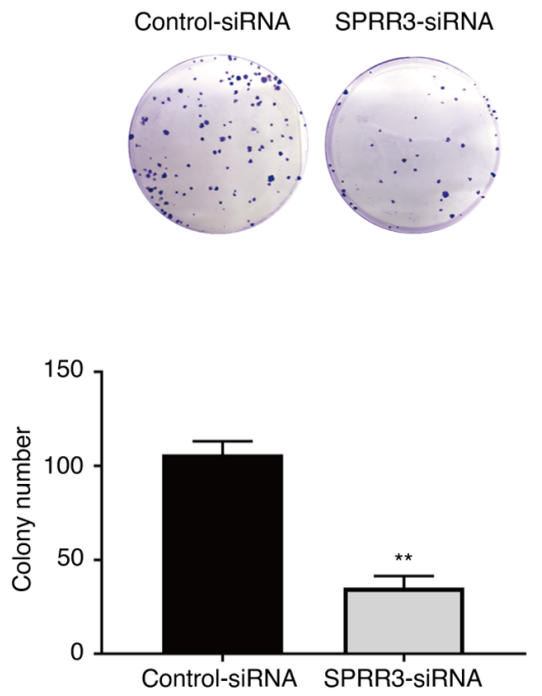
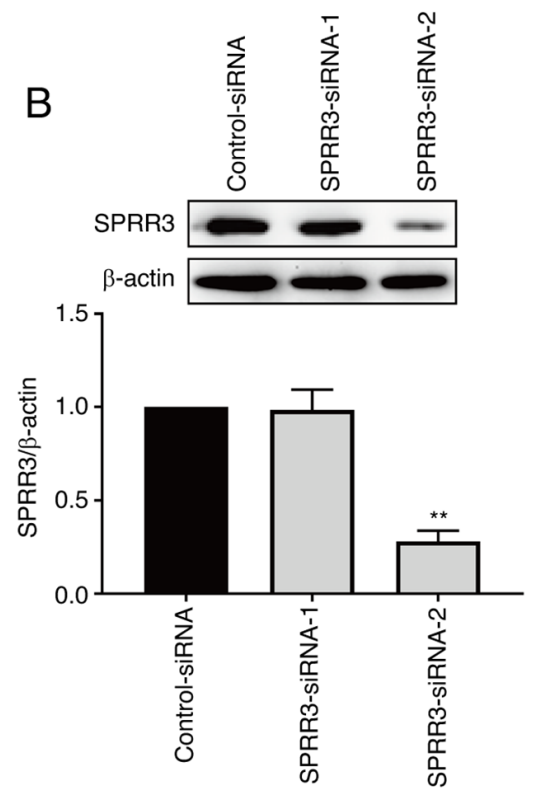

$\mathrm{E}$

E Control-siRnA
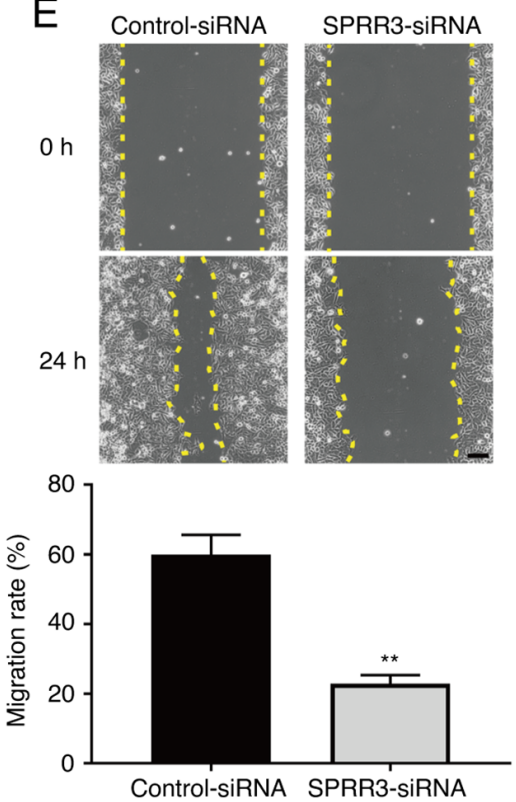

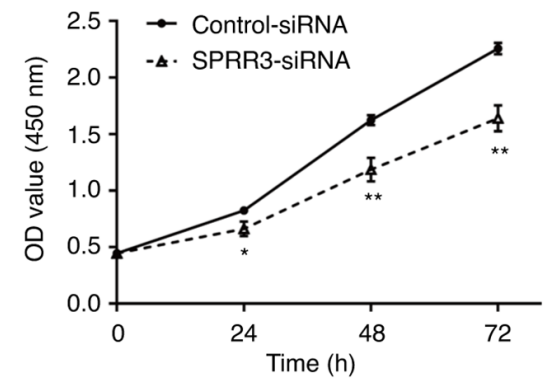

C

$\mathrm{F}$
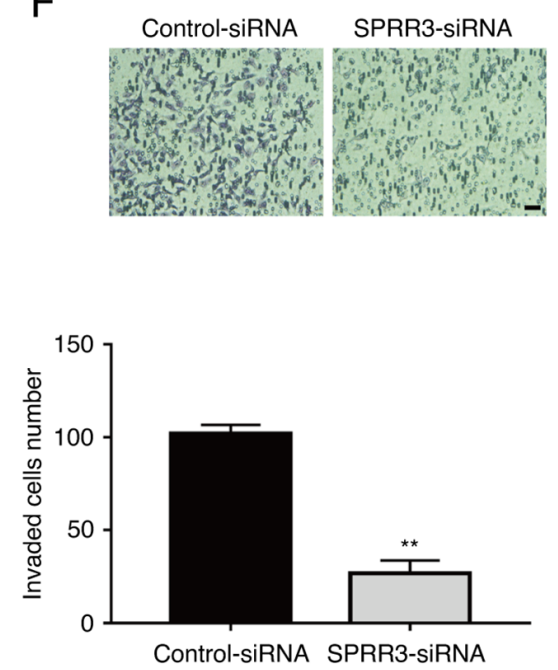

Figure 2. Role of SPRR3 in the tumor phenotypes of ccRCC cells. Screening of effective siRNAs against SPRR3 using (A) reverse transcription-quantitative PCR and (B) western blotting. The effect of SPRR3 knockdown on ccRCC cell proliferation, as detected using (C) Cell Counting Kit-8 assay and (D) colony formation assay. (E) Effects of SPRR3 knockdown on ccRCC cell migration, as detected using wound healing assay (yellow dashed lines denote the wound edge; scale bar, $100 \mu \mathrm{m}$ ). (F) Effects of SPRR3 knockdown on ccRCC cell invasion, as detected using Transwell invasion assay (scale bar, $50 \mu \mathrm{m}$ ). ${ }^{*} \mathrm{P}<0.05$ and ${ }^{* *} \mathrm{P}<0.01$ vs. control-siRNA. SPRR3, small proline-rich repeat protein 3; ccRCC, clear cell renal cell carcinoma; siRNA, small interfering RNA.

Colony formation assay. A plate colony formation assay was used to assess the proliferation of 786-O cells. The cells were plated in 12-well plates at a density of 200 cells/well. When needed, IGF-1 was used at a concentration of $100 \mathrm{ng} / \mathrm{ml}$. After incubation for 14 days at $37^{\circ} \mathrm{C}$, the colonies were washed with PBS, fixed with methanol for $10 \mathrm{~min}$ at $-20^{\circ} \mathrm{C}$ and stained with $0.5 \%$ Crystal Violet Stain Solution (Shanghai Yeasen Biotechnology Co., Ltd.) for $10 \mathrm{~min}$ at room temperature, and the number of colonies ( $>50$ cells/colony) was counted using ImageJ software.

Wound healing assay. A wound healing assay was used to assess 786-O migration. The cells were cultured in a 12-well culture plate in DMEM containing 10\% FBS until the confluence reached $100 \%$. A $200 \mu \mathrm{l}$ pipette tip was used to create a scratch in the middle of each well. The medium was replaced with serum-free DMEM. When needed, IGF-1 was used at a concentration of $100 \mathrm{ng} / \mathrm{ml}$. Images were captured of three random fields of view using a light microscope at 0 and $24 \mathrm{~h}$. Wound area was measured using ImageJ software. The results were presented as migration rate $(\%)=($ initial wound area-wound area at $24 \mathrm{~h}$ )/initial wound area $\mathrm{x} 100$.

Transwell invasion assay. Matrigel (BD Biosciences) was applied to Transwell plates (24-well, $8 \mu \mathrm{m}$; Millicell; MilliporeSigma) for $30 \mathrm{~min}$ at $37^{\circ} \mathrm{C}$ for precoating. The cells $\left(6 \times 10^{4}\right)$ were seeded in the upper chambers of the Transwell plates and incubated in serum-free DMEM, while the lower chambers were supplied with DMEM containing 


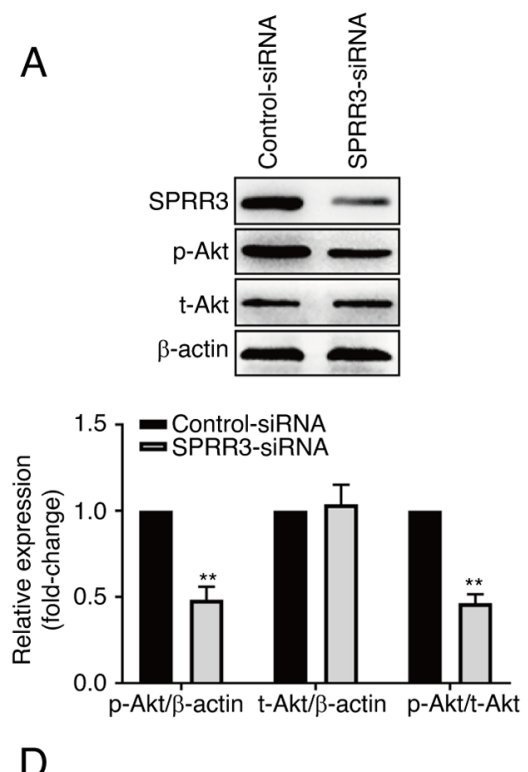

B

C
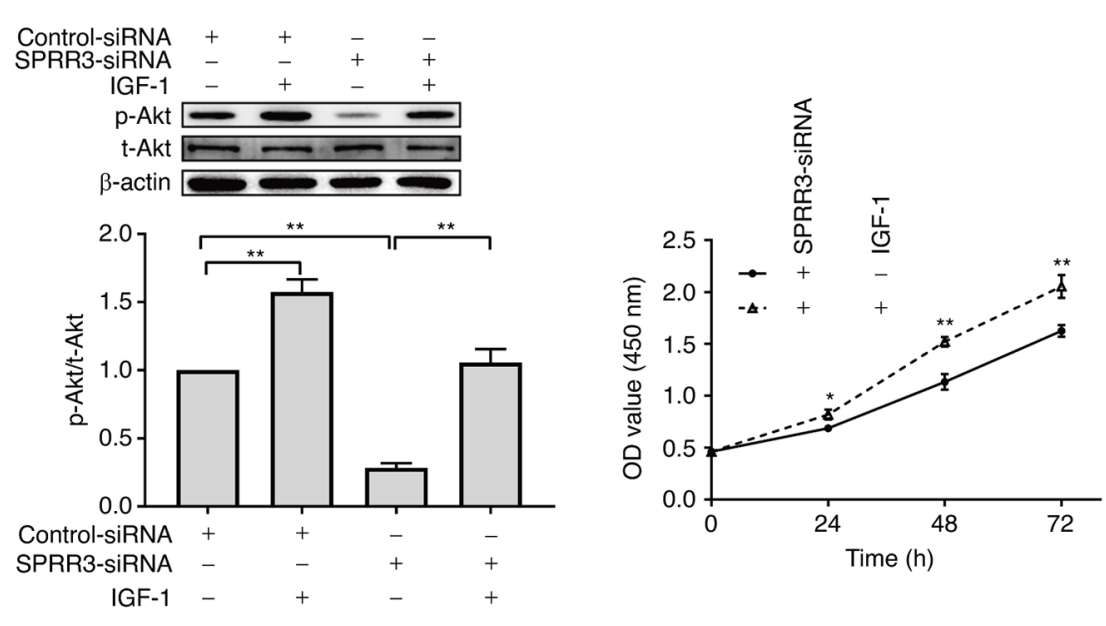

D

E

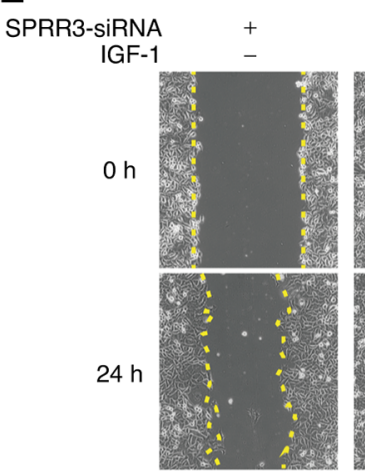

$\mathrm{F}$
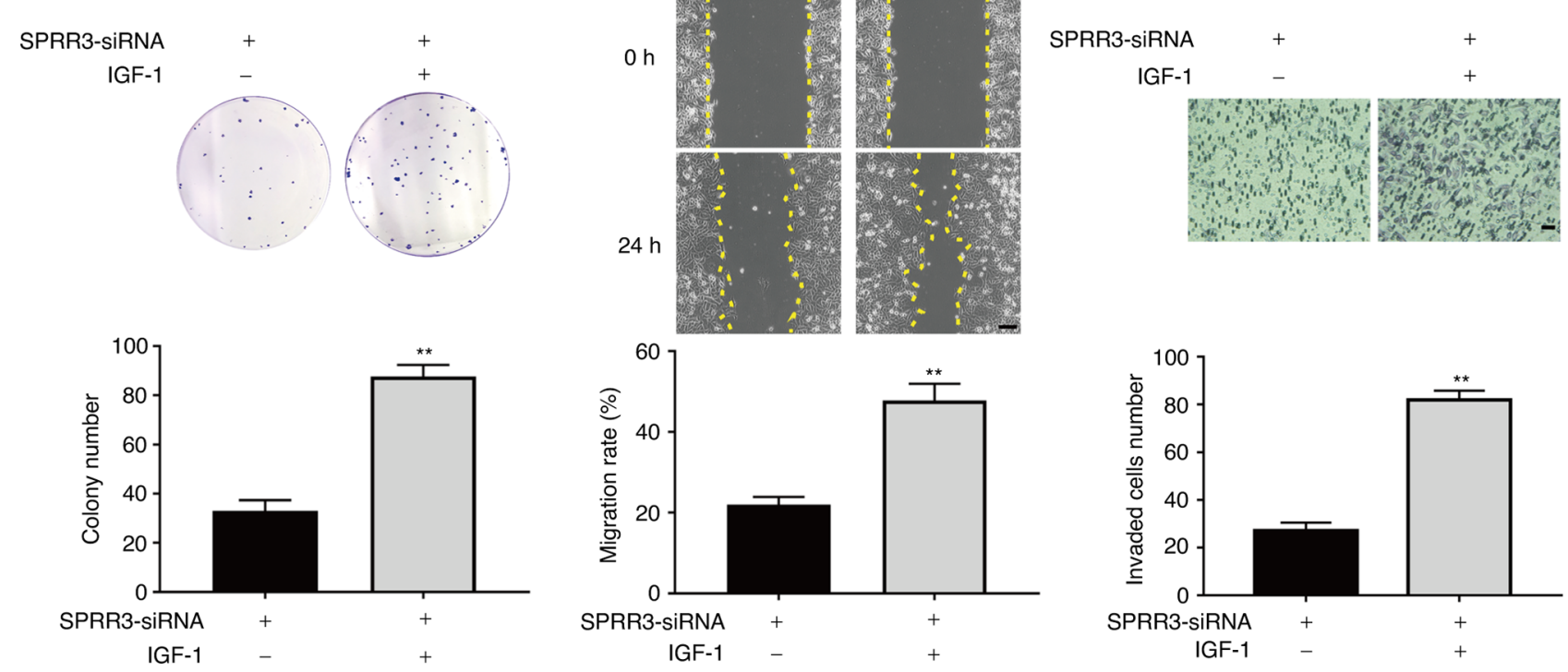

Figure 3. Involvement of the PI3K/Akt pathway in SPRR3-mediated regulation of ccRCC cells. (A) Effects of SPRR3 knockdown on the PI3K/Akt pathway, as detected using western blotting. (B) Confirming the validity of IGF-1 as an agonist of the PI3K/Akt pathway, as detected using western blotting. Effects of the PI3K/Akt pathway activation on SPRR3 knockdown-induced inhibition of ccRCC cell proliferation, as detected using (C) Cell Counting Kit-8 assay and (D) colony formation assay. (E) Effects of the PI3K/Akt pathway activation on SPRR3 knockdown-induced inhibition of ccRCC cell migration, as detected using wound healing assay (yellow dashed lines denote the wound edge; scale bar, $100 \mu \mathrm{m}$ ). (F) Effects of the PI3K/Akt pathway activation on SPRR3 knockdown-induced inhibition of ccRCC cell invasion, as detected using Transwell invasion assay (scale bar, $50 \mu \mathrm{m}$ ). ${ }^{*} \mathrm{P}<0.05$ and ${ }^{* *} \mathrm{P}<0.01$ vs. corresponding control. SPRR3, small proline-rich repeat protein 3; ccRCC, clear cell renal cell carcinoma; siRNA, small interfering RNA; p-, phosphorylated; t-, total; OD, optical density.

$10 \%$ FBS. When needed, IGF-1 was used at a concentration of $100 \mathrm{ng} / \mathrm{ml}$. After incubation for $24 \mathrm{~h}$ at $37^{\circ} \mathrm{C}$, non-invading cells on the upper face of the membrane were removed with a cotton swab, and the invaded cells were fixed with methanol for $10 \mathrm{~min}$ at $-20^{\circ} \mathrm{C}$ and stained with Giemsa dye solution (Yuanye Biotech, Co., Ltd.) for $20 \mathrm{~min}$ at room temperature. The images of each well were captured at three random fields of view using a light microscope, and the number of cells was counted using Image J software.

Luciferase reporter gene assay. The luciferase reporter plasmids of wild-type SPRR3 3'UTR (Luc-WT) or mutant SPRR3
3'UTR (Luc-Mut) were constructed using pEZX-MT06 (Guangzhou iGene Biotechnology Co., Ltd.). Empty vector, Luc-WT and Luc-Mut were co-transfected with control-mimics or miR-338-3p-mimics into 786-O cells seeded in 96-well plates using Lipofectamine ${ }^{\circledR} 3000$ transfection reagent. After $48 \mathrm{~h}$ of transfection, the cells were lysed. The results were determined using the Luc-Pair ${ }^{\mathrm{TM}}$ Duo-Luciferase HS Assay kit (Guangzhou iGene Biotechnology Co., Ltd.). The data were normalized by comparison with Renilla luciferase activity.

Statistical analysis. The quantified data were expressed as mean \pm standard deviation. Data were analyzed using one-way 
A
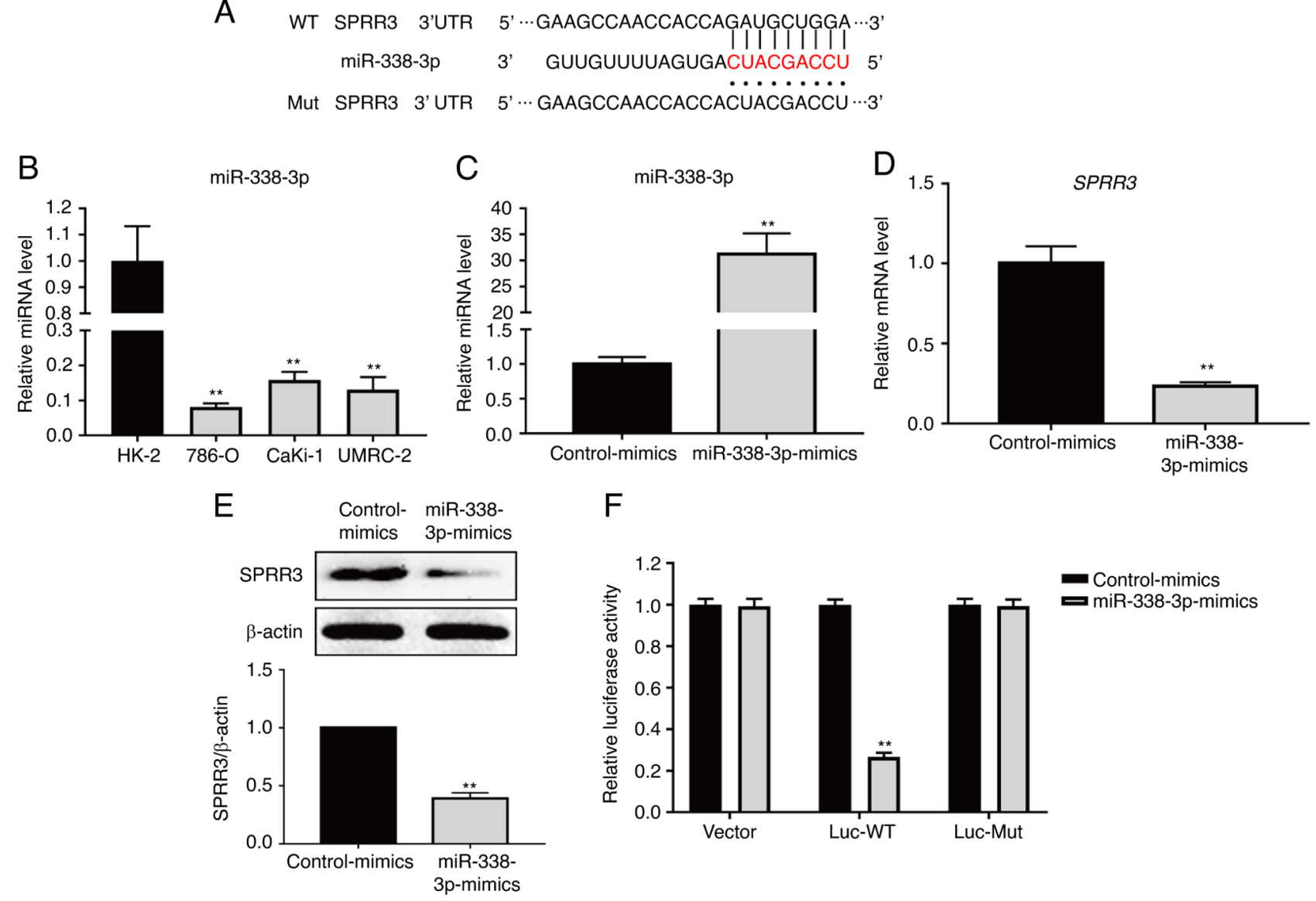

$\mathrm{F}$

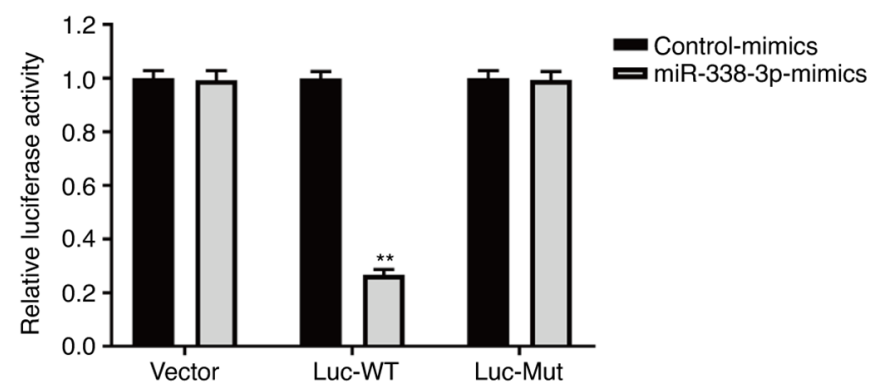

Figure 4. Relationship between miR-338-3p and SPRR3 in ccRCC cells. (A) Sequence alignment of miR-338-3p with SPRR3 3'UTR-WT and SPRR3 3'UTR-Mut. (B) miR-338-3p expression in HK-2, 786-O, CaKi-1 and UMRC-2 cell lines, as detected using RT-qPCR. (C) Confirming the validity of miR-338-3p mimics transfection, as detected using RT-qPCR. Effects of miR-338-3p overexpression on the SPRR3 expression in ccRCC cells, as detected using (D) RT-qPCR and (E) western blotting. (F) Relative luciferase activities were detected by the dual-luciferase reporter assay system. ${ }^{* *} \mathrm{P}<0.01$ vs. corresponding control. RT-qPCR, reverse transcription-quantitative PCR; SPRR3, small proline-rich repeat protein 3; ccRCC, clear cell renal cell carcinoma; miR, microRNA; UTR, untranslated region; WT, wild-type; Mut, mutated.

ANOVA followed by post hoc Dunnet's or Sidak's test, and unpaired Student's t-test with GraphPad Prism statistical package (version 7.00; GraphPad Software Inc.). $\mathrm{P}<0.05$ was considered to indicate a statistically significant difference. All experiments were repeated at least twice to verify the trends.

\section{Results}

$S P R R 3$ is a risk factor for the survival of patients with $c c R C C$ that is upregulated in ccRCC cell lines. To evaluate the relationship between SPRR3 expression and the prognosis of ccRCC, a survival analysis was performed using the UALCAN website based on the TCGA-KIRC database (containing 531 samples). Patients with low or medium SPRR3 expression levels had improved prognosis compared with those with high SPRR3 expression levels $(\mathrm{P}=0.0017$; Fig. 1A). To further evaluate the SPRR3 expression levels in ccRCC, RT-qPCR and western blotting were performed to detect the mRNA and protein levels of SPRR3, respectively, in HK-2, 786-O, CaKi-1 and UMRC-2 cell lines. The mRNA levels of SPRR3 were significantly higher in ccRCC cell lines compared with that in the normal renal cell line HK-2 (Fig. 1B). The results of western blotting were consistent with those of RT-qPCR (Fig. 1C). Overall, these data suggested that SPRR3 was a risk factor for ccRCC and was upregulated in ccRCC cell lines.

$S P R R 3$ promotes the proliferation, migration and invasion of ccRCC cells. To evaluate the role of SPRR 3 in determining the tumor phenotypes of ccRCC cells, the effects of SPRR3 knockdown on the proliferation, migration and invasion of 786-O cells were measured. The SPRR3-siRNA was initially transfected into 786-O cells to knockdown SPRR3 expression. RT-qPCR and western blotting were performed to screen for the effective siRNAs, and the results showed that SPRR3-siRNA-2 significantly decreased the mRNA and protein levels of SPRR3 (Fig. 2A and B). Therefore, siRNA-2 was selected to be used in all subsequent experiments. Next, the results of the CCK- 8 assay indicated that the viability of 786-O cells was significantly decreased in the knockdown group compared with the control group at each time point (Fig. 2C). The results of the colony formation assay demonstrated that the colony number of 786-O cells in the knockdown group was significantly decreased compared with that in the control group (Fig. 2D). The results of the wound healing assay indicated that the migration rate of 786-O cells in the knockdown group was significantly decreased compared with that in the control group (Fig. 2E). The Transwell invasion assay 
A
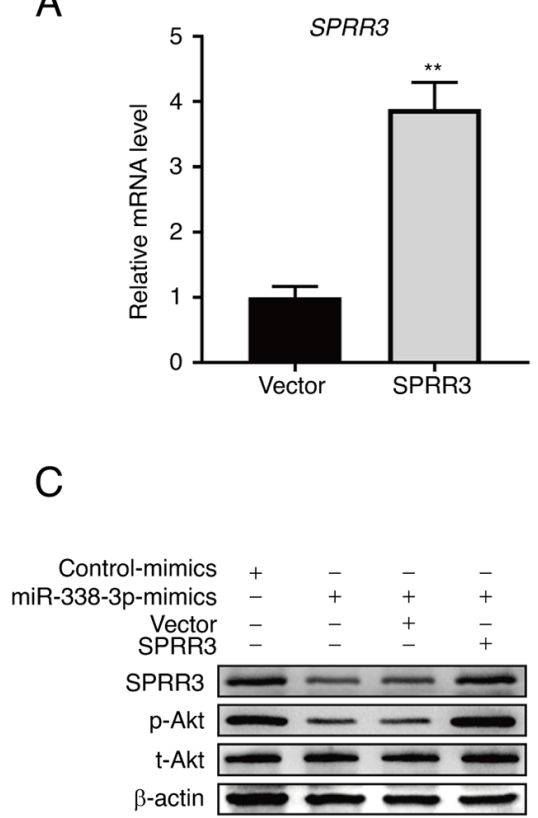

E
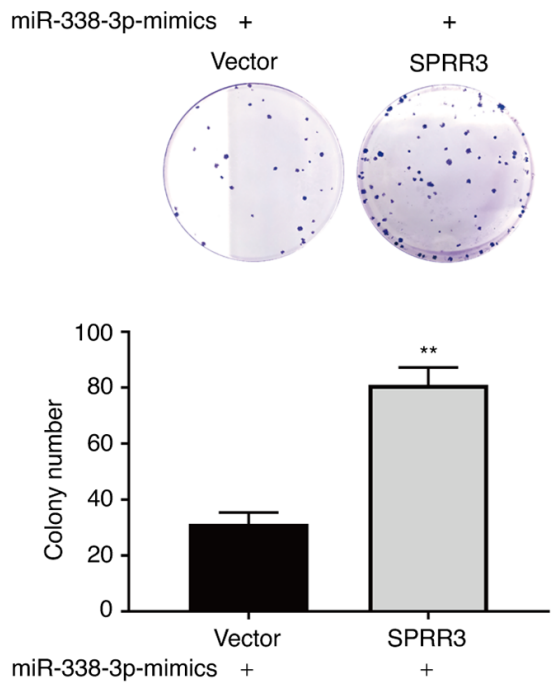

B
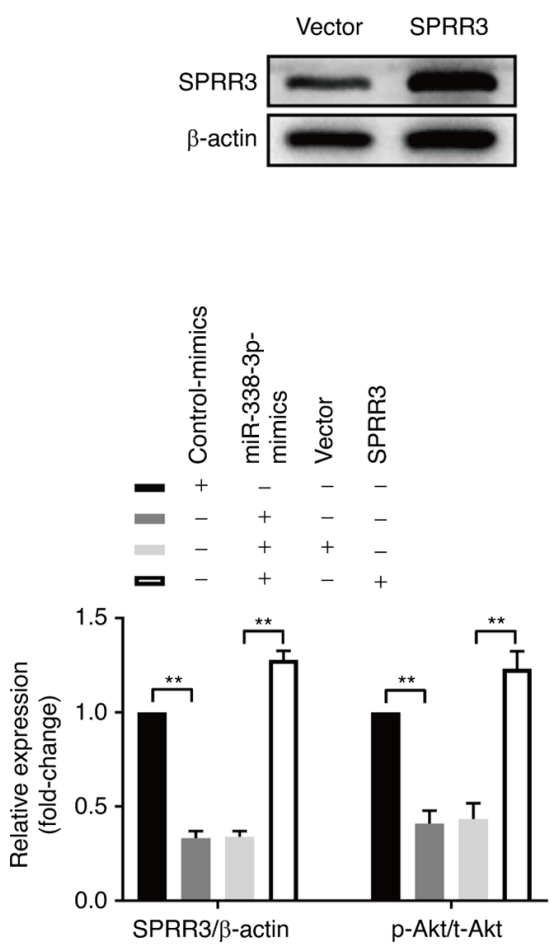

D
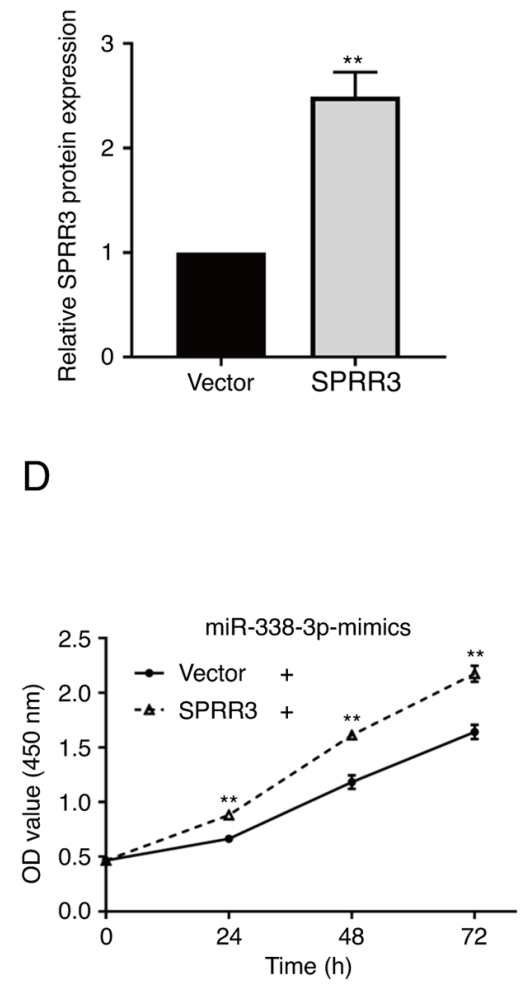

$\mathrm{F}$

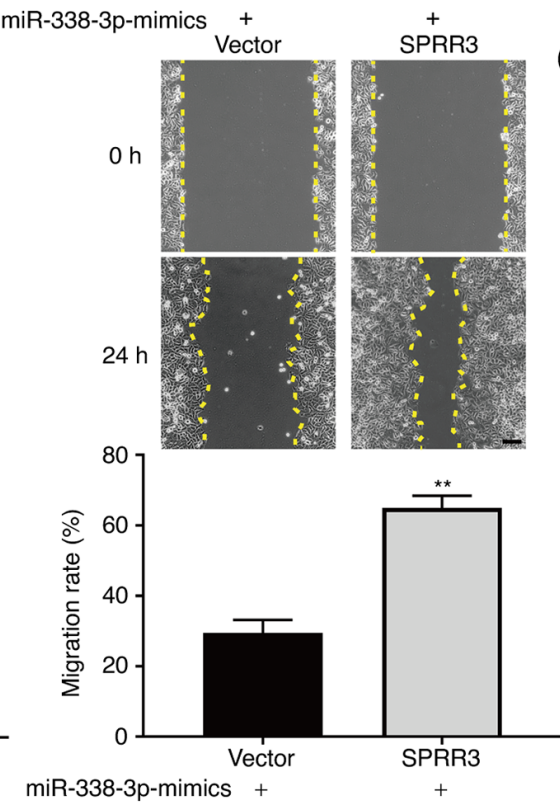

G
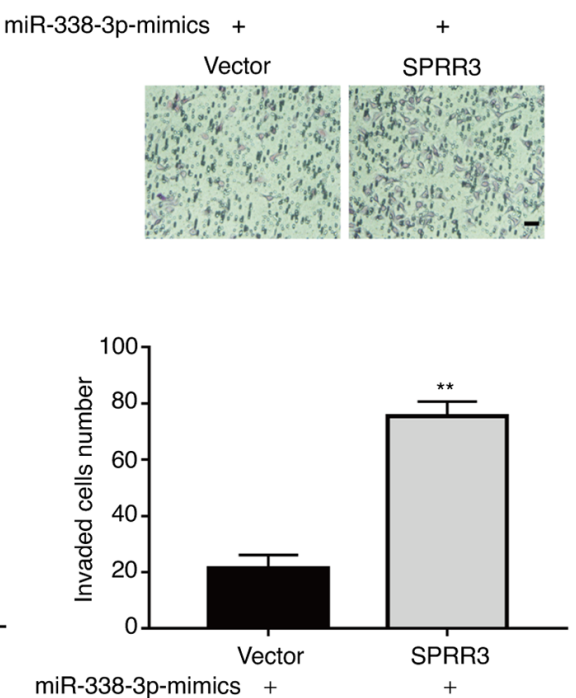

Figure 5. Role of miR-338-3p in SPRR3-mediated regulation of ccRCC cells. Confirming the validity of SPRR3 overexpression plasmid, as detected using (A) reverse transcription-quantitative PCR and (B) western blotting. (C) Effects of upregulating miR-338-3p on the PI3K/Akt pathway, and effects of SPRR3 overexpression on this regulation, as detected using western blotting. Effects of SPRR3 overexpression on the upregulation of miR-338-3p-induced inhibition of ccRCC cell proliferation, as detected using (D) Cell Counting Kit-8 assay and (E) colony formation assay. (F) Effects of SPRR3 overexpression on upregulation of miR-338-3p-induced inhibition of ccRCC cell migration, as detected using wound healing assay (yellow dashed lines denote the wound edge; scale bar, $100 \mu \mathrm{m}$ ). (G) Effects of SPRR3 overexpression on the upregulation of miR-338-3p-induced inhibition of ccRCC cell invasion, as detected using Transwell invasion assay (scale bar, $50 \mu \mathrm{m}$ ). ${ }^{* *} \mathrm{P}<0.01$ vs. corresponding control. SPRR3, small proline-rich repeat protein 3; ccRCC, clear cell renal cell carcinoma; miR, microRNA; p-, phosphorylated; t-, total; OD, optical density.

indicated that the number of invaded 786-O cells per field in the knockdown group was significantly decreased compared with that in the control group (Fig. 2F). These data demonstrated that SPRR3 promoted the proliferation, migration and invasion of ccRCC cells.
SPRR3 regulates the tumor phenotypes of ccRCC cells via the PI3K/Akt pathway. To further explore the downstream signaling pathway responsible for theSPRR3-regulated phenotypes of ccRCC cells, the present study investigated whether the PI3K/Akt pathway was involved in this regulation. The 


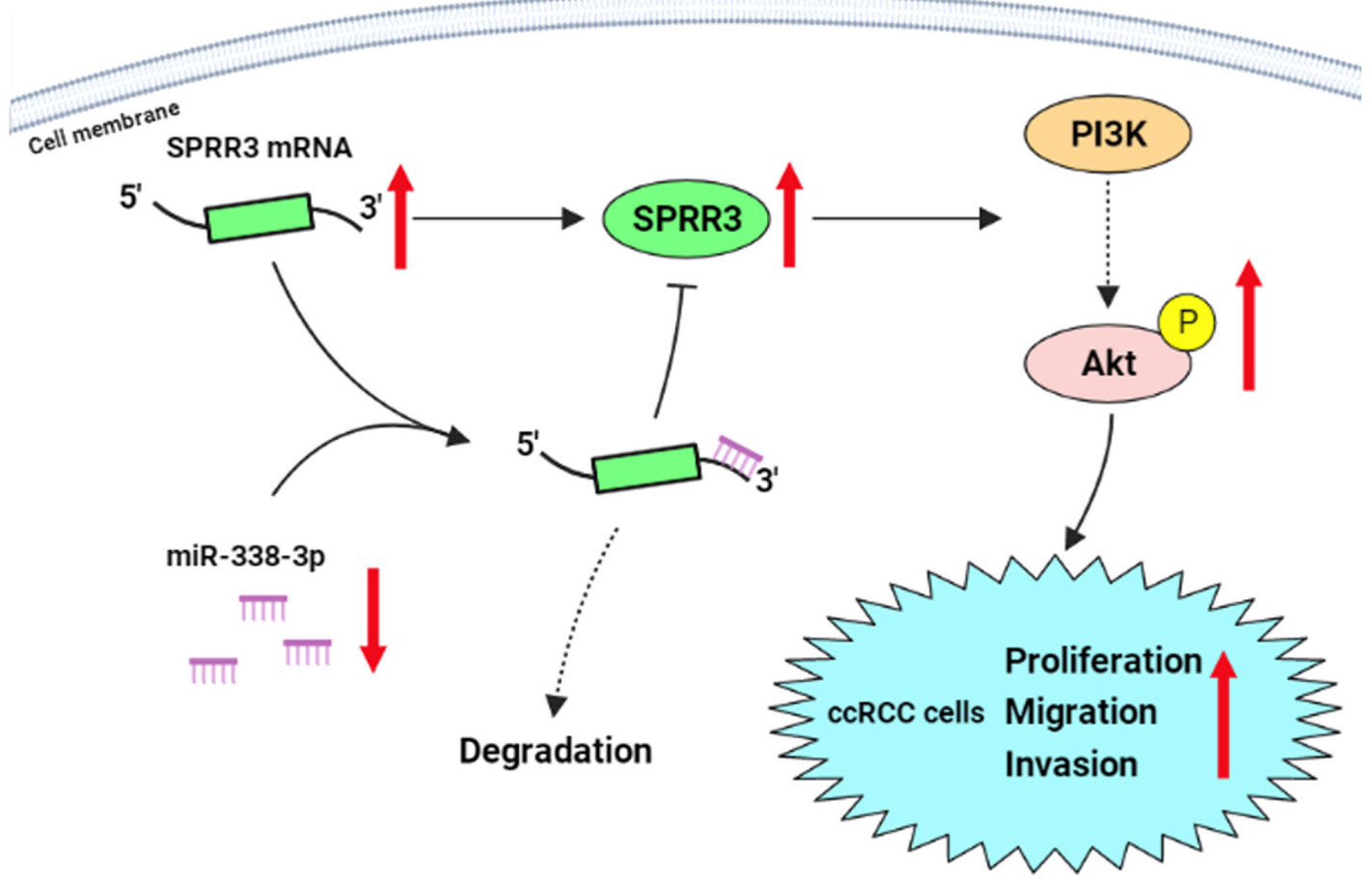

Figure 6. Schematic illustration of the regulatory mechanism of miR-338-3p/SPRR3 axis via the PI3K/Akt pathway in ccRCC cells. Figure created with BioRender.com. miR, microRNA; ccRCC, clear cell renal cell carcinoma; SPRR3, small proline-rich repeat protein 3.

level of phosphorylation of Akt, measured based on the ratio of phosphorylated Akt (p-Akt)/total Akt (t-Akt), was used as an indicator of the activation status of the PI3K/Akt pathway. The effect of SPRR3 knockdown on the PI3K/Akt pathway was initially examined. As presented in Fig. 3A, compared with the control group, the level of p-Akt was significantly decreased, and no significant change was observed in the level of t-Akt in the knockdown group. The ratio of p-Akt/t-Akt was significantly decreased in the knockdown group, suggesting that SPRR3 knockdown inhibited the PI3K/Akt pathway in 786-O cells. Next, IGF-1, a known agonist of the PI3K/Akt pathway, was used to verify whether this signaling pathway was involved in SPRR3-regulated 786-O cell proliferation, migration and invasion. As presented in Fig. 3B, IGF-1 significantly increased the level of $\mathrm{p}$-Akt/t-Akt compared with the control group, and significantly reversed the decreased level of p-Akt/t-Akt led by SPRR3 knockdown, thus confirming the validity of IGF-1. The results of the CCK- 8 assay demonstrated that activation of the PI3K/Akt pathway significantly reversed the SPRR3 knockdown-induced reduction in the viability of 786-O cells at each time point (Fig. 3C). The results of the colony formation assay indicated that activation of the PI3K/Akt pathway significantly reversed the SPRR3 knockdown-induced decrease in the colony number of 786-O cells (Fig. 3D). The results of the wound healing assay showed that activation of the PI3K/Akt pathway significantly reversed the SPRR3 knockdown-induced decrease in the migration rate of 786-O cells (Fig. 3E). The results of the Transwell invasion assay showed that activation of the PI3K/Akt pathway significantly reversed the SPRR3 knockdown-induced decrease in invaded 786-O cells per field (Fig. 3F). Overall, these data demonstrated that SPRR3 regulated the tumor phenotypes of ccRCC cells via the PI3K/Akt pathway.
miR-338-3p directly targets SPRR3 and negatively regulates SPRR3 expression. To explore the upstream regulator of SPRR3, TargetScan was used to predict the potential functional miRNAs. The binding site between SPRR3 mRNA 3'UTR and miR-338-3p was predicted using TargetScan (Fig. 4A). The expression levels of miR-338-3p in $\mathrm{HK}-2$, 786-O, CaKi-1 and UMRC-2 cell lines were determined using RT-qPCR, which revealed that the levels of miR-338-3p were significantly lower in ccRCC cell lines compared with HK-2 cells (Fig. 4B). Transfection of miRNA mimics for miR-338-3p overexpression was used to verify its association with SPRR3 in 786-O cells. As presented in Fig. 4C, the RT-qPCR results showed that the level of miR-338-3p was significantly increased in the mimics group compared with the control group, which confirmed that the miR-338-3p-mimics were effective. The mRNA and protein levels of SPRR3 were further investigated, which revealed that the expression levels of SPRR3 mRNA and protein were significantly decreased in the mimic groups compared with those in the control group (Fig. 4D and E), which indicated that miR-338-3p inhibited SPRR3 expression in 786-O cells. WT and mut-type SPRR3 3'UTR luciferase reporter vectors were used to perform a dual-luciferase reporter assay to confirm the binding relationship and position between SPRR3 mRNA and miR-338-3p (Fig. 4A). As presented in Fig. 4F, in the Luc-WT transfection group, the relative luciferase activity of 786-O cells transfected with miR-338-3p-mimics was significantly lower compared with that of cells transfected with control-mimics; however, no significant change was observed in the Luc-Mut transfection groups. These data demonstrated that miR-338-3p directly targeted SPRR3 and negatively regulated SPRR3 expression in ccRCC cells. 
miR-338-3p inhibits the PI3K/Akt pathway and tumor phenotypes of ccRCC cells by downregulating SPRR3 expression. Based on the aforementioned findings, the present study further investigated whether miR-338-3p inhibited the PI3K/Akt pathway and the tumor phenotypes of ccRCC cells by downregulating SPRR3. The overexpression efficiency of SPRR3 plasmids was validated using RT-qPCR and western blotting, and the results showed that the mRNA and protein levels of SPPR3 were significantly increased in the overexpression group (Fig. 5A and B). The p-Akt/t-Akt ratio was significantly decreased in the miR-338-3p-mimics group compared with that in the control group, which confirmed that miR-338-3p inhibited the PI3K/Akt pathway in 786-O cells (Fig. 5C). Rescue experiments were carried out. SPRR3 overexpression significantly reversed the downregulation of SPRR3 by the miR-338-3p-mimics, under which downstream pathway and tumor phenotypes regulated by the miR-338-3p-mimics were observed. As presented in Fig. 5C, SPRR3 overexpression significantly reversed the reduction of $\mathrm{p}-\mathrm{Akt} / \mathrm{t}-\mathrm{Akt}$ by the miR-338-3p-mimics. Additionally, the results of the CCK-8 assay showed that the overexpression of SPRR 3 significantly reversed the miR-338-3p-mimics-induced inhibition of 786-O cell proliferation (Fig. 5D). The results of the colony formation assay showed that the overexpression of SPRR3 significantly reversed the miR-338-3p-mimics-induced decrease in the colony number of 786-O cells (Fig. 5E). The results of the wound healing assay showed that the overexpression of SPRR3 significantly reversed the miR-338-3p-mimics-induced decrease in the migration rate of 786-O cells (Fig. 5F). The results of the Transwell invasion assay showed that SPRR3 overexpression significantly reversed the miR-338-3p-mimics-induced decrease in the number of invaded cells per field (Fig. 5G). These data demonstrated that miR-338-3p inhibited the PI3K/Akt pathway and the tumor phenotypes of ccRCC cells by downregulating SPRR3.

\section{Discussion}

To the best of our knowledge, this study demonstrated the role of SPRR3 in determining the tumor phenotypes of ccRCC cells for the first time, and further uncovered its associated downstream and upstream mechanisms. SPRR3 as a tumor promoter has been revealed to be upregulated in some types of cancer. SPRR3 accelerates the proliferation and invasion of colorectal cancer cells (4), the proliferation of breast cancer cells (5), the proliferation and invasion of glioblastoma multiforme cells (6) and the proliferation and invasion of non-small-cell lung cancer cells (7). These results are similar to those reported in the present study, which suggested the expression of SPRR3 as a poor prognostic factor and a potential novel therapeutic target for ccRCC. By contrast, several studies on esophageal cancer have revealed that low expression of SPRR3 is associated with disease progression $(8,22,23)$. These findings indicate that the role of SPRR3 may vary in different cancer types. Additionally, both loricrin and SPRR3 are cornified cell envelope precursor proteins, and their genes locates in the same cluster on chromosome 1q21 (24). Loricrin is reported to be associated with tumor metastatic spread. This may indicate that loricrin is involved in SPRR3-mediated regulation in ccRCC (25). However, the role of SPRR3 in other diseases still needs to be explored further.

miR-338-3p is downregulated in RCC (26), which was also confirmed by the ccRCC cell lines in the present study. Furthermore, previous studies have uncovered several downstream molecules involved in miR-338-3p-mediated inhibition of tumor phenotypes in ccRCC cells. Tong et al (12) revealed that miR-338-3p inhibits the proliferation and invasion of 786-O and Caki-1 cells by targeting SOX-4. Yang et al (13) revealed that miR-338-3p inhibits the proliferation, migration and invasion of ccRCC cells by downregulating the expression of ETS1. Zhu et al (14) revealed that CAV-1 is also a downstream target of miR-338-3p in ccRCC cells. The present study demonstrated that SPRR3 was a novel direct target of miR-338-3p, and that miR-338-3p inhibited the proliferation, migration and invasion of ccRCC cells by downregulating SPRR3. In addition to these findings, more research is needed to investigate the how the downstream mechanism of miR-338-3p involved in the development of ccRCC. The association between miR-338-3p and survival of patients with ccRCC also warrants further investigation.

The aberrant activation of the PI3K/Akt signaling pathway promotes the proliferation, migration and invasion of ccRCC cells (15). SPRR3 activates the PI3K/Akt pathway in colorectal and breast cancer cells $(4,5)$. Additionally, SPRR3 activates this pathway in vascular smooth muscle cells and cardiac fibroblasts $(9,10)$. miR-338-3p inhibits the PI3K/Akt pathway in RCC (16), which is consistent with the results of the present study showing miR-338-3p-mediated inhibition of the PI3K/Akt pathway in 786-O cells. Moreover, miR-338-3p exerts its anti-tumor effects by inhibiting the PI3K/Akt pathway in breast cancer and neuroblastoma cells $(27,28)$. The present study demonstrated that the PI3K/Akt pathway was involved in the regulation of ccRCC cell tumor phenotypes via the miR-338-3p/SPRR3 axis, which presented a new model of the mechanism driving ccRCC cell development (Fig. 6). Based on these findings, it was hypothesized that this regulation may occur in other tumors, although this needs to be verified. Additionally, further in vivo animal experiments are needed to verify the present findings.

In conclusion, the current study demonstrated that the upregulation of SPRR3 played a promotive role in the proliferation, migration and invasion of ccRCC cells in vitro, which was directly regulated by the downregulation of miR-338-3p; moreover, the activation of the PI3K/Akt pathway was involved in this regulation. This finding not only enriches the understanding of the mechanism understanding ccRCC development, but also presents a potential novel therapeutic target for this disease.

\section{Acknowledgements}

Not applicable.

\section{Funding}

This study was supported by the PhD Research Project of Jilin Engineering Normal University (grant no. BSKJ201923). 


\section{Availability of data and materials}

The datasets used and/or analyzed during the current study are available from the corresponding author on reasonable request.

\section{Authors' contributions}

LW and XL conceived and designed the study. MW and QG performed the experiments, which were guided and supervised by LW and XL. MW and XL performed the data analysis. MW completed the first draft of the manuscript. LW and XL revised the final version of the manuscript. MW, $\mathrm{XL}$ and $\mathrm{LW}$ confirm the authenticity of all the raw data. All authors read and approved the final manuscript.

\section{Ethics approval and consent to participate}

Not applicable.

\section{Patient consent for publication}

Not applicable.

\section{Competing interests}

The authors declare that they have no competing interests.

\section{References}

1. Bray F, Ferlay J, Soerjomataram I, Siegel R, Torre L and Jemal A: Global cancer statistics 2018: GLOBOCAN estimates of incidence and mortality worldwide for 36 cancers in 185 countries. CA Cancer J Clin 68: 394-424, 2018.

2. Powles T, Albiges L, Staehler M, Bensalah K, Dabestani S, Giles RH, Hofmann F, Hora M, Kuczyk MA, Lam TB, et al: Updated European association of urology guidelines: Recommendations for the treatment of first-line metastatic clear cell renal cancer. Eur Urol 73: 311-315, 2018.

3. Ljungberg B, Bensalah K, Canfield S, Dabestani S, Hofmann F, Hora M, Kuczyk MA, Lam T, Marconi L, Merseburger AS, et al: EAU guidelines on renal cell carcinoma: 2014 update. Eur Urol 67: 913-924, 2015.

4. Cho DH, Jo YK, Roh SA, Na YS, Kim TW, Jang SJ, Kim YS and Kim JC: Upregulation of SPRR3 promotes colorectal tumorigenesis. Mol Med 16: 271-277, 2010.

5. Kim JC, Yu JH, Cho YK, Jung CS, Ahn SH, Gong G, Kim YS and Cho DH: Expression of SPRR3 is associated with tumor cell proliferation in less advanced stages of breast cancer. Breast Cancer Res Treat 133: 909-916, 2012.

6. Liu Q, Zhang C, Ma G and Zhang Q: Expression of SPRR3 is associated with tumor cell proliferation and invasion in glioblastoma multiforme. Oncol Lett 7: 427-432, 2014.

7. Li Q, Wang $\mathrm{Y}, \mathrm{Hu} \mathrm{R}$ and Yang G: Dysregulation of SPR R3/miR-876-3p axis contributes to tumorigenesis in non-small-cell lung cancer. Onco Targets Ther 13: 2411-2419, 2020.

8. Zhang Y, Feng YB, Shen XM, Chen BS, Du XL, Luo ML, Cai Y, Han YL, Xu X, Zhan QM, et al: Exogenous expression of Esophagin/SPRR3 attenuates the tumorigenicity of esophageal squamous cell carcinoma cells via promoting apoptosis. Int J Cancer 122: 260-266, 2008.

9. Segedy AK, Pyle AL, Li B, Zhang Y, Babaev VR, Jat P, Fazio S, Atkinson JB, Linton MF and Young PP. Identification of small proline-rich repeat protein 3 as a novel atheroprotective factor that promotes adaptive Akt signaling in vascular smooth muscle cells. Arterioscler Thromb Vasc Biol 34: 2527-2536, 2014.
10. Saraswati S, Lietman C, Li B, Mathew S, Zent R and Young P: Small proline-rich repeat 3 is a novel coordinator of PDGFR $\beta$ and integrin $\beta 1$ crosstalk to augment proliferation and matrix synthesis by cardiac fibroblasts. FASEB J 34: 7885-7904, 2020.

11. Harrandah A, Mora R and Chan E: Emerging microRNAs in cancer diagnosis, progression, and immune surveillance. Cancer Lett 438: 126-132, 2018

12. Tong Z, Meng X, Wang J and Wang L: MicroRNA-338-3p targets SOX 4 and inhibits cell proliferation and invasion of renal cell carcinoma. Exp Ther Med 14: 5200-5206, 2017.

13. Yang X, Zhang Y and Fan H: Downregulation of SBF2-AS1 functions as a tumor suppressor in clear cell renal cell carcinoma by inhibiting miR-338-3p-targeted ETS1. Cancer Gene Ther 28: 813-827, 2020.

14. Zhu Q, Zhan D, Zhu P, Chong Y and Yang Y: CircAKT1 acts as a sponge of miR-338-3p to facilitate clear cell renal cell carcinoma progression by up-regulating CAV1. Biochem Biophys Res Commun 532: 584-590, 2020

15. Peng X, Yang J, Qiang Y, Sun R, Cao Y, Zheng LS, Peng LX, Lang YH, Mei Y, Li CZ, et al: PTPN3 inhibits the growth and metastasis of clear cell renal cell carcinoma via inhibition of PI3K/AKT signaling. Mol Cancer Res 18: 903-912, 2020.

16. Li G, Chong T, Yang J, Li H and Chen $\mathrm{H}$ : Kinesin motor protein KIFC1 is a target protein of miR-338-3p and is associated with poor prognosis and progression of renal cell carcinoma. Oncol Res 27: 125-137, 2018.

17. Chandrashekar DS, Bashel B, Balasubramanya SAH, Creighton CJ, Ponce-Rodriguez I, Chakravarthi BVSK and Varambally S: UALCAN: A portal for facilitating tumor subgroup gene expression and survival analyses. Neoplasia 19: 649-658, 2017.

18. Cancer Genome Atlas Research Network: Comprehensive molecular characterization of clear cell renal cell carcinoma. Nature 499: 43-49, 2013.

19. Agarwal V, Bell G, Nam J and Bartel D: Predicting effective microRNA target sites in mammalian mRNAs. Elife 4: e05005, 2015.

20. Wang L, Sui M and Wang X: miR-338-3p suppresses the malignancy of T-cell lymphoblastic lymphoma by downregulating HOXA3. Mol Med Rep 20: 2127-2134, 2019.

21. Livak KJ and Schmittgen TD: Analysis of relative gene expression data using real-time quantitative PCR and the 2(-Delta Delta C(T)) method. Methods 25: 402-408, 2001

22. Chen BS, Wang MR, Cai Y, Xu X, Xu ZX, Han YL and Wu M: Decreased expression of SPRR3 in Chinese human oesophageal cancer. Carcinogenesis 21: 2147-2150, 2000.

23. de A Simão T, Souza-Santos PT, de Oliveira DS, Bernardo V, Lima SC, Rapozo DC, Kruel CD, Faria PA, Ribeiro Pinto LF and Albano RM: Quantitative evaluation of SPRR3 expression in esophageal squamous cell carcinoma by qPCR and its potential use as a biomarker. Exp Mol Pathol 91: 584-589, 2011.

24. Hohl D, de Viragh P, Amiguet-Barras F, Gibbs S, Backendorf C and Huber M: The small proline-rich proteins constitute a multigene family of differentially regulated cornified cell envelope precursor proteins. J Invest Dermatol 104: 902-909, 1995.

25. Ellis R, Tang D, Nasr B, Greenwood A, McConnell A, Anagnostou ME, Elias M, Verykiou S, Bajwa D, Ewen T, et al: Epidermal autophagy and beclin 1 regulator 1 and loricrin: A paradigm shift in the prognostication and stratification of the American joint committee on cancer stage I melanomas. Br J Dermatol 182: 156-165, 2020.

26. Huang Y, Wu Y, Zeng L, Shan W and Huang L: The tumor suppressor role of microRNA-338-3p in renal cell carcinoma. Oncol Lett 16: 2195-2200, 2018.

27. He J, Wang J, Li S, Li T, Chen K and Zhang S: Hypoxia-inhibited miR-338-3p suppresses breast cancer progression by directly targeting ZEB2. Cancer Sci 111: 3550-3563, 2020.

28. Xu Z, Sun Y, Wang D, Sun H and Liu X: SNHG16 promotes tumorigenesis and cisplatin resistance by regulating miR-338-3p/PLK4 pathway in neuroblastoma cells. Cancer Cell Int 20: 236, 2020.

This work is licensed under a Creative Commons Attribution-NonCommercial-NoDerivatives 4.0 International (CC BY-NC-ND 4.0) License. 